\title{
Microdeletions Including FMR1 in Three Female Patients with Intellectual Disability - Further Delineation of the Phenotype and Expression Studies
}

\author{
A.M. Zink ${ }^{a} \quad$ E. Wohlleber ${ }^{a} \quad$ H. Engels ${ }^{a} \quad$ O.K. Rødningen ${ }^{f} \quad$ K. Ravn ${ }^{g}$ \\ S. Heilmann ${ }^{a, b} \quad$ J. Rehnitz ${ }^{d} \quad$ N. Katzorke ${ }^{d} \quad$ C. Kraus $^{\text {e }}$ S. Blichfeldt ${ }^{\text {h }}$ \\ P. Hoffmann ${ }^{a, b, j} \quad$ H. Reutter ${ }^{a, c}$ F.F. Brockschmidt ${ }^{a, b} \quad$ M. Kreiß-Nachtsheim ${ }^{a}$

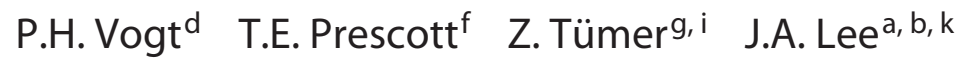 \\ ${ }^{a}$ Institute of Human Genetics, ${ }^{b}$ Department of Genomics, Life \& Brain Center, and ${ }^{c}$ Department of Neonatology, \\ Children's Hospital, Rheinische Friedrich-Wilhelms-University, Bonn, ${ }^{d}$ Molecular Genetics and Infertility Unit, \\ Department of Gynecology, Endocrinology and Reproductive Medicine, University Women Hospital, Heidelberg, \\ and ${ }^{\mathrm{e}}$ Institute of Human Genetics, Friedrich-Alexander-University, Erlangen-Nuremberg, Germany; ${ }^{\mathrm{f} D e p a r t m e n t}$ of \\ Medical Genetics, Oslo University Hospital, Oslo, Norway; ${ }^{9}$ Applied Human Molecular Genetics, Kennedy Center, \\ and hediatric Department L55, Glostrup University Hospital, Glostrup, i'Department of Cellular and Molecular

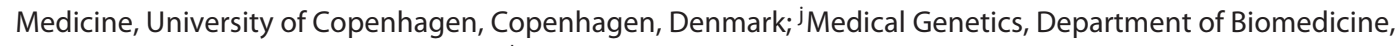 \\ University Hospital, Basel, Switzerland; ' Greenwood Genetic Center, Greenwood, S.C., USA
}

\section{Key Words}

Expression · FMR1 - Intellectual disability · Microdeletion · Xq27.3-q28

\begin{abstract}
Fragile $X$ syndrome (FXS) is one of the most common causes of intellectual disability/developmental delay (ID/DD), especially in males. It is caused most often by CGG trinucleotide repeat expansions, and less frequently by point mutations and partial or full deletions of the FMR1 gene. The wide clinical spectrum of affected females partly depends on their Xinactivation status. Only few female ID/DD patients with microdeletions including FMR1 have been reported. We describe 3 female patients with 3.5-, 4.2- and 9.2-Mb de novo microdeletions in Xq27.3-q28 containing FMR1. X-inactivation was random in all patients, yet they presented with ID/ DD as well as speech delay, macrocephaly and other features
\end{abstract}

\section{KARGER}

() 2014 S. Karger AG, Basel

$1661-8769 / 14 / 0052-0065 \$ 39.50 / 0$

E-Mail karger@karger.com

www.karger.com/msy attributable to FXS. No signs of autism were present. Here, we further delineate the clinical spectrum of female patients with microdeletions. FMR1 expression studies gave no evidence for an absolute threshold below which signs of FXS present. Since FMR1 expression is known to be highly variable between unrelated females, and since FMR1 mRNA levels have been suggested to be more similar among family members, we further explored the possibility of an intrafamilial effect. Interestingly, FMR1 mRNA levels in all 3 patients were significantly lower than in their respective mothers, which was shown to be specific for patients with microdeletions containing FMR1.

(c) 2014 S. Karger AG, Basel

A.M.Z., E.W., H.E., T.E.P., Z.T., and J.A.L. contributed equally to this work. 
Fragile X syndrome (FXS) is one of the most common causes of hereditary intellectual disability (ID) and has a prevalence of approximately $1 / 4,500$ males and 1/9,000 females [O'Donnell and Warren, 2002]. The clinical spectrum of FXS in males typically includes moderate intellectual disability/developmental delay (ID/DD), characteristic dysmorphisms such as a large head, long face, protruding ears, and large testes after puberty, as well as behavioral abnormalities. Affected females with FXS present with highly variable clinical features ranging from almost all of the physical features described in males to mild learning disabilities without further signs, depending in part on their X-chromosome inactivation (XCI) status [de Vries et al., 1996].

While full-length CGG expansions ( $\geq 200$ repeats) in the $5^{\prime}$-untranslated region of the fragile X mental retardation gene FMR1 are the most common cause of FXS (>99\%), other etiological mechanisms, such as partial or full deletions of FMR1 as well as point mutations, are rare [Hammond et al., 1997]. Consequently, there is a paucity in the literature regarding the clinical picture for small genomic rearrangements involving FMR1 [Nagamani et al., 2012]. To date, only 7 female index patients with deletions harboring FMR1, ranging from 2.7 to $10.6 \mathrm{Mb}$ in size, have been reported. ID/DD is common to all 7 patients. Four of these patients also present with obesity or are borderline overweight, and clinical features of the FXS spectrum are generally present. FMR 1 has been discussed as a dosage-sensitive gene necessary for normal brain function and development, but the pathologic mechanism of the deletions remains unclear [Nagamani et al., 2012]. As described for female FXS patients with CGG repeat expansions, the severity of the phenotype for females with FMR1 deletions also correlates with their XCI status [Dahl et al., 1995; Wolff et al., 1997; Probst et al., 2007].

A correlation between FMR1 expression and the number of CGG repeats has been well described for males with FXS. Full-length CGG repeat expansions cause hypermethylation of the FMR1 promoter and the loss of both FMR1 mRNA and protein expression [Feng et al., 1995; Allen et al., 2004]. For patients with alleles within the premutation range (55-200 repeats), FMR1 transcript levels are elevated and increase with increasing repeat length [Tassone et al., 2000; Allen et al., 2004; García-Alegría et al., 2007].

In females, however, the relationship between FMR1 transcript levels and CGG repeat length is more complex, as it mirrors that observed for males only after accounting for the XCI status [Allen et al., 2004; García-Alegría et al.,
2007]. In general, FMR1 transcript levels vary considerably between females [Allen et al., 2004; Schuettler et al., 2011]. However, there is preliminary evidence suggesting a possible intrafamilial effect, with mRNA levels being more similar among members of the same family than among unrelated females [García-Alegría et al., 2007]. This might, in part, explain the differences in FMR1 mRNA levels observed between unrelated healthy females.

In this study, we present 3 unrelated female patients with heterozygous overlapping deletions in Xq27.3-q28 harboring the FMR1 gene. In addition to delineating further the phenotype of females with Xq27.3-q28 microdeletions, we also elucidate the role of FMR1 gene expression in the etiology of our patients' phenotypes. Moreover, we explore the above-mentioned notion of an intrafamilial effect regarding FMR1 transcript levels by examining the relative FMR1 expression levels in patients with microdeletions and their mothers compared to control daughter-mother pairs. Our data indicate that FMR1 transcript levels, relative to that of a closely-related female control (e.g. the mother), might be correlated with the presence and possibly even the severity of an FXS phenotype in females with FMR1 deletions.

\section{Clinical Reports}

Patient 1

Patient 1 , a girl aged 16 years and 10 months, was the third child born to non-consanguineous German parents with an unremarkable family history and 2 healthy older brothers. After an uneventful pregnancy, the birth was at term but complicated by umbilical cord enlacement. Birth weight was 4,620 g (97th centile), length 56 $\mathrm{cm}$ (97th centile) and occipitofrontal head circumference (OFC) $36 \mathrm{~cm}$ (90th centile). At the age of 3 months, the parents' suspicion of delayed development was confirmed by a pediatrician, and subsequently, developmental milestones were delayed. She walked independently at 25 months. Her first words were spoken at approximately 18 months and she started using whole sentences at 3 years. At 5 years of age, her scores on the Denver Developmental Scales corresponded to an age of 3-3.5 years. Severe muscular hypotonia, particularly of the face, resulting in hypersalivation was noted in childhood. At the age of 8 years and 3 months, she started psychotherapy for behavioral problems and exhibited passivity. Puberty proceeded normally, and menarche occurred at about 14.5 years. Echocardiography, karyotype (400-500 band resolution), basic neurometabolic work-up (plasma and urinary amino acids, urinary mucopolysaccharides, and organic acids), as well as DNAbased analyses for Prader-Willi syndrome and maternal UPD 14 yielded normal results.

Upon examination at the age of 16 years and 10 months, her weight was $113 \mathrm{~kg}$ ( $>97$ th centile), height $168 \mathrm{~cm}$ (50-75th centile), and OFC $57.5 \mathrm{~cm}$ ( $>97$ th centile). She had mild ID and muscular
66

Mol Syndromol 2014;5:65-75

DOI: $10.1159 / 000357962$
Zink et al. 
hypotonia. Her obesity was ascribed to hyperphagia and lacking the feeling of satiety. Her social interaction was adequate. She was subtly dysmorphic with a long face, slight hypertelorism, a bulbous nasal tip, and a prominent chin, and had mild neurodermatitis. Tapering fingers, mildly shortened 5 th fingers and mild joint hyperextensibility of fingers and hands were noted. The 4th and 5th toes appeared foreshortened, possibly due to short metatarsals, and had hypoplastic nails (fig. $1 \mathrm{a}-\mathrm{c}$ ). The parents declined X-ray investigations of the hands and feet.

\section{Patient 2}

Patient 2 was the 3.5-year-old fifth child born to non-consanguineous Norwegian parents with an unremarkable family history. During pregnancy, vaginal bleeding prompted antenatal ultrasound examinations which revealed normal fetal growth and movements. Delivery at term was by emergency Caesarean section due to breech presentation complicated by umbilical cord prolapse. Her birth weight was $3,735 \mathrm{~g}$ (75th centile), crown-rump length $35 \mathrm{~cm}$ (50-97.5th centile), and OFC $35 \mathrm{~cm}$ (50th centile). Neonatally, there were no medical complications. However, in contrast to her 4 older sisters, she slept poorly, cried excessively and initially accepted comforting only from her mother. Generalized hypotonia, tongue protrusion, tactile defensiveness, and chronic vomiting contributed to feeding being a major medical issue for the first 2.5 years of life. She was hospitalized several times before the age of 2 years because of severe constipation and was treated surgically for intestinal obstruction at 14 months. Intestinal biopsy did not confirm the clinical suspicion of Hirschsprung's disease. At the age of 1.5 years, no cause was found for elevated serum liver enzymes and bilirubin in the absence of hepatosplenomegaly. Mild proteinuria and hematuria were noted at around 2 years of age. She was intermittently catheterized for approximately 8 months starting at the age of 2.5 years because of high residual urine volumes indicative of a neurogenic bladder. Ultrasound investigation of the urinary tract and magnetic resonance imaging (MRI) of the spine revealed no abnormalities.

Motor development was slow but within normal limits until the age of 9 months when she regressed transiently after contracting chicken pox. She sat at 15 months, crawled at 16 months and walked at 2 years and 7 months. At the age of 3.5 years, she had 40 single words and 60 signs, and preferred signing to speaking. Her expressive language at this time was judged to be at the 19-month level, while language comprehension was at the 4-year level (Psychoeducational Profile, PEP-3). At that time, she asked for food on awakening at night and may have had a diminished sense of satiety.

She was referred for genetic counseling at 3.5 years after the detection of a microdeletion by diagnostic work-up for DD (see Results). She had a round full face but was not dysmorphic (fig. 1d). Her facial features resembled those of her 4 older healthy sisters. Her finger joints were hyperextensible. Her height was $101 \mathrm{~cm}$ (50-75th centile), weight was $18.4 \mathrm{~kg}$ (90-97th centile), and OFC was $53 \mathrm{~cm}(\sim 1 \mathrm{~cm}>97$ th centile). She was clumsy in gross motor activities, but had good fine motor skills.

The extensive diagnostic work-up included, among other investigations, conventional karyotyping, cerebral MRI, ophthalmological examination, urinary screening for metabolic disease, abdominal ultrasound, as well as standard and 24-hour electroencephalography. All results were normal.
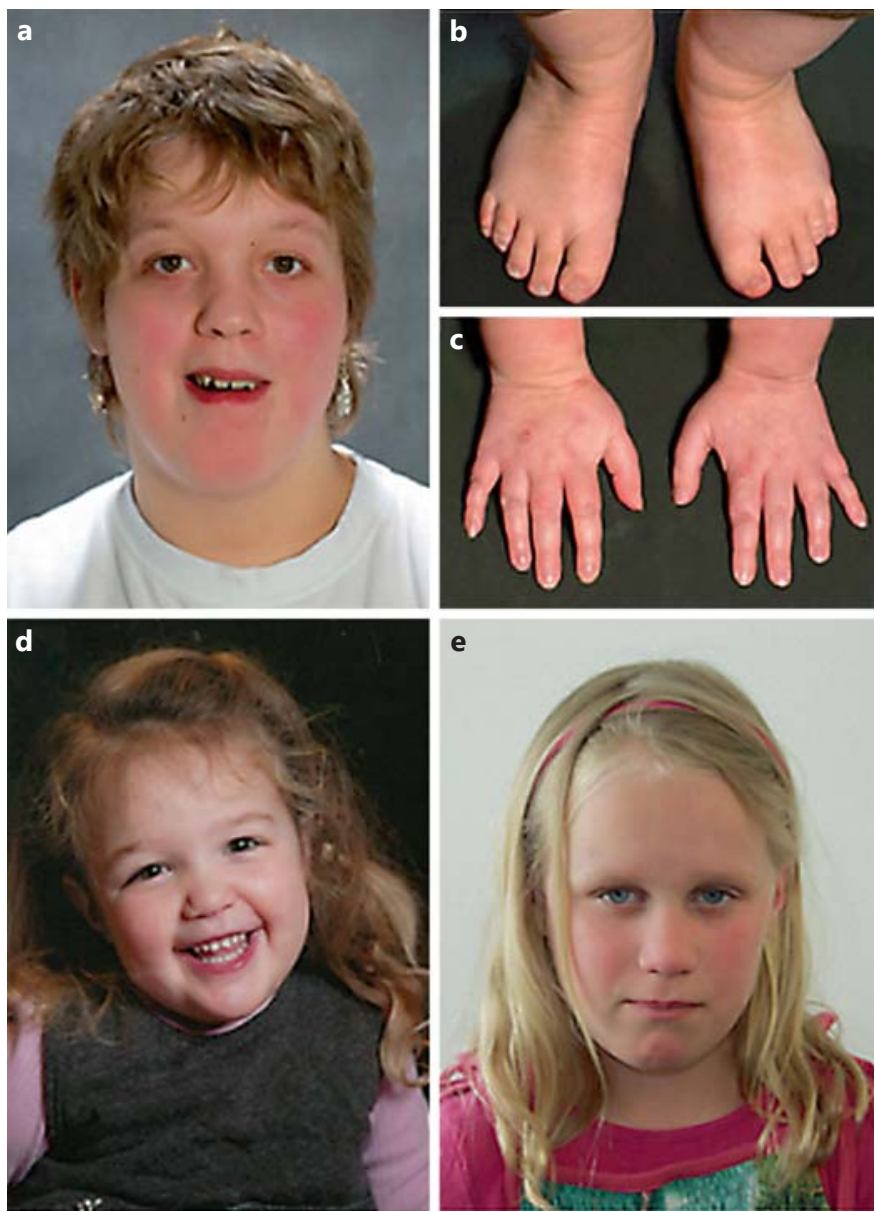

Fig. 1. Appearance of patients 1,2 and 3. a-c Patient 1 at age 16 years and 10 months. a Frontal view: long face, bulbous nasal tip and prominent chin. b Feet: hypoplastic nails and shortened appearance of 4th and 5th toes. c Hands: slightly tapering fingers, slightly shortened 5th fingers. d Patient 2. Frontal view at age 3.5 years: full cheeks, high forehead. e Patient 3. Frontal view at age 9 years: high forehead, slightly deep-set eyes.

\section{Patient 3}

Patient 3, a 9-year-old girl (fig. 1e), was the first-born child of non-consanguineous, healthy Danish parents. Her 2 younger siblings were healthy, and the family history was unremarkable. After an uneventful pregnancy, she was born with normal measurements: weight 3,500 g (50-75th centile) and length $53 \mathrm{~cm}(50-75$ th centile); OFC was not reported. The neonatal period was uneventful. Developmental delay was recognized at 2 years when a speech therapist diagnosed a speech delay of about 9-12 months. Motor development was only slightly delayed, and she sat and walked at appropriate ages. At the age of 5 years and 8 months, the results of a Wechsler Preschool and Primary Scale of Intelligence (WPPSI) test corresponded to an age of 3 years.

When she was 6 years and 2 months old, her height was $116 \mathrm{~cm}$ ( 25 th centile) and her weight was $27 \mathrm{~kg}$ (50-70th centile). Ophthalmological evaluation and audiometric tests gave normal results. 
Neurological examination as well as muscular tonus and reflexes were normal. Besides deep-set eyes, she exhibited no dysmorphisms.

At the age of 8.5 years, her speech was slightly delayed and she attended a special class for children with DD. She recognized almost all capital letters, but could not read lower-case writing. Her fine and gross motor skills were normal and her social interaction very good. She tired quickly and could not concentrate for long periods of time.

Prior to DNA array analysis, an extensive diagnostic work-up including metabolic parameters such as lysosomal enzymes, TSH, calcium and phosphate levels, screening of urine, and cytogenetic analyses gave normal results.

\section{Materials and Methods}

\section{Genomic Analyses}

Molecular karyotyping and confirmation by independent methods were performed in the respective study centers. All positions are according to GRCh37/hg19. Primer sequences are available upon request.

Patient 1. Genomic DNA of patient 1 was analyzed using an Illumina Sentrix HumanHap550-Duo v3 BeadChip (Illumina, San Diego, Calif., USA) according to the manufacturer's protocol. CNV calling and quantitative PCR (qPCR) to confirm the deletion and to demonstrate its de novo occurrence was performed as published previously [Engels et al., 2009]. Patient and parental samples were analyzed with 5 amplicons (amplicon 1, chrX:145,682,259-145,682,374; amplicon 2, chrX:147,111, 821-147,111,929; amplicon 3, chrX:147,616,487-147,616,598; amplicon 4, chrX:147,022,741-147,022,869; amplicon 5, chrX:145, $255,536-145,255,654)$.

Patient 2. Genomic DNA was labeled according to the manufacturer's instructions using Genomic DNA Labeling Kit PLUS (Agilent Technologies Inc., Palo Alto, Calif., USA), and array comparative genomic hybridization $(\mathrm{CGH})$ was performed using $\mathrm{Hu}-$ man Genome CGH Microarray 44K (Agilent Technologies Inc.). Data were analyzed using Agilent Feature Extraction v9.1 software and plotted according to chromosomal location using Agilent DNA Analytics v4.0 software. Array CGH analysis of the mother and FMR1 repeat analysis of the father were performed to demonstrate the de novo occurrence of the deletion.

Patient 3. Array CGH was carried out using CytoChip BAC array v2.0.1 (BlueGnome Ltd., Cambridge, UK). Multiplex ligationdependent probe amplification (MLPA) was performed using a commercial kit (P106; MRC-Holland, The Netherlands) containing probes for FMR1 and AFF2 (formerly FMR2) to demonstrate the de novo occurrence of the deletion. Deletion breakpoints were finely mapped by qPCR using SYBR ${ }^{\circledR}$-Green I detection chemistry and a 7500 Fast Real-Time PCR System according to the manufacturer's instructions (Applied Biosystems, Foster City, Calif., USA). qPCR was also carried out to confirm the deletion in patient 3 and to demonstrate its de novo occurrence. Patient and parental sampleswereanalyzed with 3 amplicons (amplicon 1, chrX:139,585,756139,585,806; amplicon 2, chrX:148,797,702-148,797,752; amplicon 3, chrX:149,391,705-149,391,755).
FMR1 Sequence and Repeat Analyses

The FMR1 gene was sequenced in the index patients. Primer sequences are available upon request. The PCR products were sequenced unidirectionally using Big Dye Terminator v1.1 and run on a 3500xL Genetic Analyzer (Applied Biosystems). DNA sequences were compared to reference sequence NG_007529.1. FMR1 CGG repeat lengths were analyzed using standard diagnostic procedures [Fernandez-Carvajal et al., 2009].

\section{$X$-Inactivation Analysis}

$\mathrm{X}$-inactivation analysis was performed according to standard methods [Lau et al., 1997]. PCR fragments were analyzed on an ABI3100 Sequencer, and the fragment sizes were determined with GeneScan and Genotyper software (Applied Biosystems).

\section{Control Daughter-Mother Pairs for Expression Analyses}

Although an ideal control group for the analysis of FMR1 expression and the observation of a possible intrafamilial effect may be age-matched healthy daughter-mother pairs, for ethical reasons we did not perform this analysis to avoid the possibility of identifying premutation alleles in asymptomatic minors. Instead, we analyzed already existing RNA samples of 3 daughter-mother pairs without ID/DD, which were ascertained because of premature ovarian failure (POF) in all daughters and 1 mother (POF2M). These control pairs were: daughter POF1 (age 32 years)/mother POF1M (63 years); POF2 (36 years)/POF2M (64 years); POF3 (22 years)/POF3M (39 years). In control daughter POF3, a microscopically visible $\mathrm{X}$-chromosomal deletion had been detected and confirmed using fluorescence in situ hybridization (FISH) with a ToTelVysion subtelomeric probe (46,X,del(X)(q27).ish $\operatorname{del}(\mathrm{X})$ (DXYS130+,DXYS224-)). She showed 100\% skewed XCI and no ID/DD or other abnormalities. qPCR was performed to confirm the deletion of FMR1 in control daughter POF3 and to exclude the deletion in the other daughter-mother pairs, as published previously [Engels et al., 2009]. Control daughter POF1 and mother POF1M are FMR1 premutation carriers (POF1: 31/79 repeats; POF1M: 29/72 repeats). FMR1 repeat expansion was excluded in the other control daughter-mother pairs. We reasoned that this control group would be suitable for observing a possible intrafamilial effect (i.e. mRNA levels being more similar among mothers and daughters than among unrelated females), if such an effect could be demonstrated regardless of the various scenarios in the control pairs: FMR1 deletion versus normal copy number, FMR1 premutation versus normal repeat length, POF in the daughter only versus POF in both mother and daughter.

\section{Expression Analyses}

Expression analyses for FMR1, IDS, AFF2, and FMR1NB were performed in the 3 patients with microdeletions (P1, P2, P3), their respective mothers (P1M: 45 years; P2M: 32 years; P3M: 38 years), and 5 healthy female control persons (ages 22-24 years), as well as for FMR1 only in an additional 3 POF daughter-mother control pairs (see above). Whole blood RNA from the 3 patients, their mothers and the 5 healthy controls was extracted using the PAXgene Blood system (Qiagen, Hilden, Germany), and from the POF controls using the RNAeasy Mini-Kit (Qiagen). Identical amounts of RNA of all patients and controls were reverse-transcribed using Superscript II (Invitrogen, Carlsbad, Calif., USA). Expression levels were measured using predesigned TaqMan ${ }^{\circledR}$ gene expression assays (Applied Biosystems; Hs00924547_m1; Hs00541336_m1; 
Table 1. Summary of features seen in 3 patients with de novo microdeletions in Xq27.3-q28

\begin{tabular}{|c|c|c|c|}
\hline & Patient 1 & Patient 2 & Patient 3 \\
\hline Age & 16 y $10 \mathrm{mo}$ & 3 y 6 mo & $9 y$ \\
\hline Array & Illumina $550 \mathrm{~K}$ & Agilent $44 \mathrm{~K}$ & CytoChip BAC array v2.0.1 \\
\hline \multicolumn{4}{|l|}{ Deletion } \\
\hline Location $^{\mathrm{a}}$ & chrX:145,126,550-148,653,916 & chrX:144,910,904-149,185,262 & chrX:139,307,660-148,548,633 \\
\hline Size, $\mathrm{Mb}$ & 3.53 & 4.27 & 9.24 \\
\hline RefSeq genes $^{\mathrm{b}}$ & & 14 & 24 \\
\hline Origin & de novo & de novo & de novo \\
\hline $\mathrm{X}$-inactivation ${ }^{\mathrm{c}}$ & $\begin{array}{l}\text { random } \\
(62: 38)\end{array}$ & $\begin{array}{l}\text { random } \\
(57: 43)\end{array}$ & $\begin{array}{l}\text { random } \\
(48: 52)\end{array}$ \\
\hline Pregnancy/birth & umbilical cord enlacement & $\begin{array}{l}\text { vaginal bleeding, umbilical cord prolapse, } \\
\text { Caesarean section }\end{array}$ & uneventful \\
\hline Psychomotor development & $\begin{array}{l}\text { delayed } \\
\text { diagnosis of developmental delay at } 3 \\
\text { mo, first words at } 18 \text { mo, unsupported } \\
\text { walking at } 25 \text { mo }\end{array}$ & $\begin{array}{l}\text { delayed } \\
\text { normal development until } 9 \text { mo, then } \\
\text { transient regression after chicken pox, } \\
\text { unsupported walking at } 31 \text { mo, } 40 \text { single } \\
\text { words at } 3 \text { y } 6 \text { mo }\end{array}$ & $\begin{array}{l}\text { delayed } \\
\text { diagnosis of psychomotor delay at } 2 \mathrm{y} \text {, } \\
\text { speech delay, mild motor } \\
\text { developmental delay }\end{array}$ \\
\hline Degree of ID & mild & mild & mild \\
\hline Growth parameters & $\begin{array}{l}\text { weight }>\mathbf{9 7} \text { th ct, height } 50-75 \text { th } \mathrm{ct} \text {, } \\
\text { OFC }>\mathbf{9 7 \text { th }} \text { ct }\end{array}$ & $\begin{array}{l}\text { weight } 90-97 \text { th ct, height } 50-75 \text { th ct, } \\
\text { OFC }>97 \text { th ct }\end{array}$ & $\begin{array}{l}6 \text { y: weight } 50-75 \text { th ct, height } 25 \text { th ct } \\
9 \text { y: weight } 50-70 \text { th ct, height } 50 \text { th ct, } \\
\text { OFC } 97 \text { th ct }\end{array}$ \\
\hline Muscle tonus & $\begin{array}{l}\text { hypotonia } \\
\text { (severe in childhood, particularly facial } \\
\text { muscles, resulting in hypersalivation) }\end{array}$ & generalized hypotonia & normal \\
\hline Dysmorphisms & $\begin{array}{l}\text { long face, mild hypertelorism, } \\
\text { bulbous nasal tip, prominent chin }\end{array}$ & full cheeks, high forehead & high forehead \\
\hline Hands/feet & $\begin{array}{l}\text { mild joint hyperextensibility of } \\
\text { fingers and hands, foreshortened } \\
\text { appearance of } 4 \text { th and } 5 \text { th toes }\end{array}$ & hyperextensibility of finger joints & n.r. \\
\hline Behavior & behavioral problems, passivity & n.r. & n.r. \\
\hline Puberty & normal & n.a. & n.r. \\
\hline Others & lacking sense of satiety & $\begin{array}{l}\text { diminished sense of satiety } \\
\text { first year of life: excessive crying, tactile } \\
\text { defensiveness, chronic vomiting/feeding } \\
\text { problems, severe constipation }\end{array}$ & n.r. \\
\hline
\end{tabular}

Bold letters $=$ Common features; $\mathrm{ct}=$ centile; $\mathrm{mo}=$ months; n.a. $=$ not applicable; n.r. $=$ not reported; $\mathrm{y}=$ years.

${ }^{a}$ All positions are according to GRCh37. ${ }^{\mathrm{b}}$ Number of deleted protein-coding RefSeq genes. ${ }^{\mathrm{c}}$ Performed on peripheral blood; analyzed locus: AR.

Hs00171400_m1; Hs00240821_m1) in triplicate, with each reaction containing $2 \mu \mathrm{l}$ of cDNA template in a $10-\mu \mathrm{l}$ reaction volume, on an ABI Prism 7900HT Fast Real-Time PCR System (Applied Biosystems). The cycling conditions were as follows: $50^{\circ} \mathrm{C}$ for $2 \mathrm{~min}$, denaturation at $95^{\circ} \mathrm{C}$ for $10 \mathrm{~min}$ followed by 40 cycles at $95^{\circ} \mathrm{C}$ for $15 \mathrm{~s}$, and a combined annealing and extension step at $60^{\circ} \mathrm{C}$ for $60 \mathrm{~s}$. Duplex reactions contained both target (FAM) and endogenous control (VIC) probes and primers. Two different endogenous controls (Human B2M (beta-2-microglobulin) Endogenous Control and Human PPIA (cyclophilin A) Endogenous Control; TaqMan ${ }^{\circledR}$ Endogenous Controls, Applied Biosystems) were assayed in parallel and in separate duplex reactions. Normal-

Deletions Including FMR1 in Female

Patients with ID ized results were compared to the results of 5 healthy female controls. Each triplicate experiment was performed twice. $p$ values were calculated using a two-sided Wilcoxon test.

\section{Results}

\section{Genomic Analyses}

Molecular karyotyping for the 3 patients revealedde novo heterozygous deletions in Xq27.3-q28. The deletion 


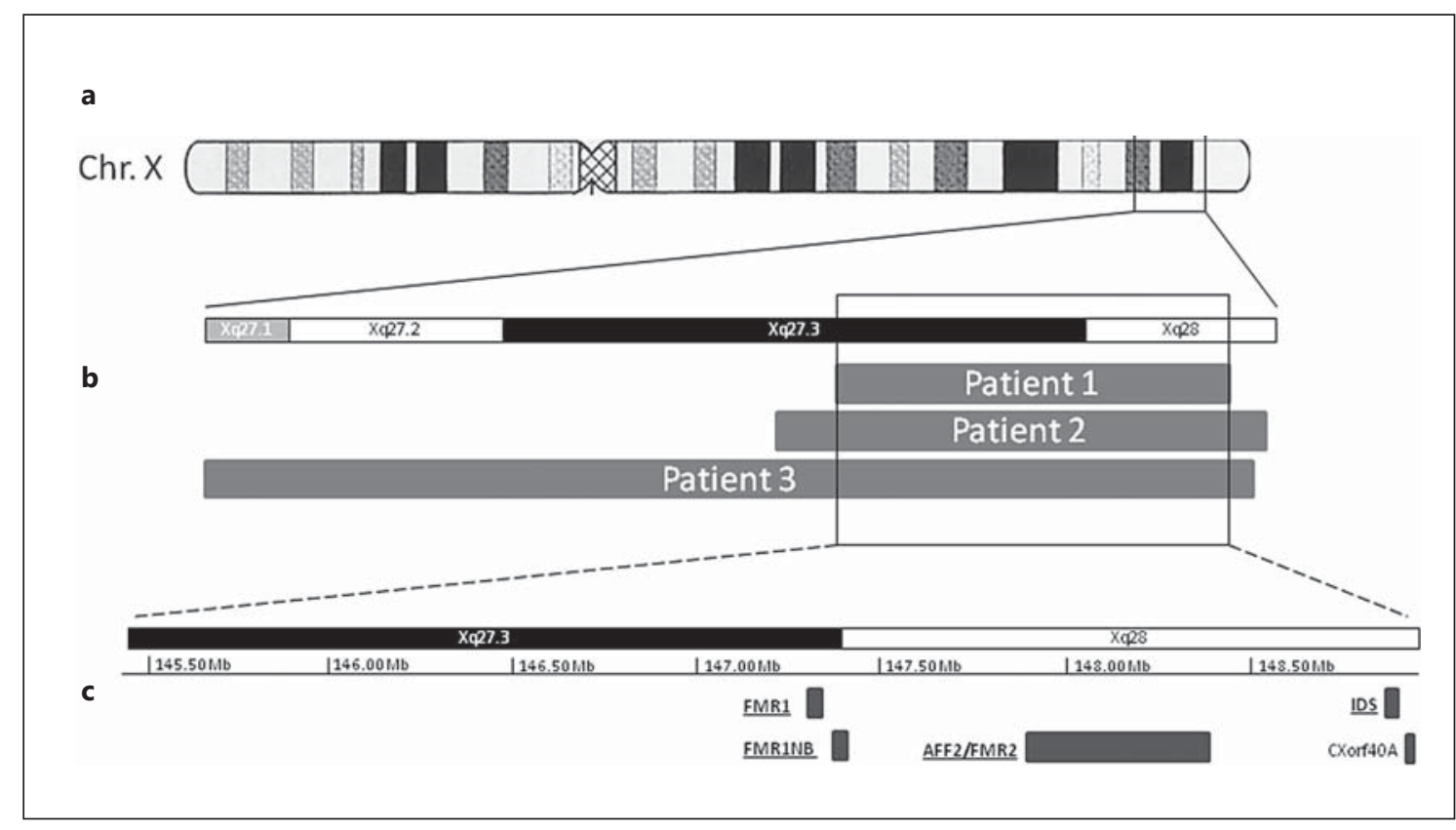

Fig. 2. Schematic representation of the Xq27-q28 deletions in patients 1, 2 and 3. a Diagram of the X chromosome. $\mathbf{b}$ Magnification of the Xq27.1-q28 region with grey bars representing the patients' deletions and dashed lines delineating the smallest region of overlap (SRO) of the deletions. c Magnification of the SRO with dark grey bars representing the 5 genes located within. Underlining marks genes for which expression studies were performed. All positions are according to GRCh37.

sizes and breakpoints are shown in detail in table 1 . The smallest region of overlap (SRO) of $3.53 \mathrm{Mb}$ corresponds to the deletion in patient 1 (start SNP: rs5965673, chrX:145,126,550; end SNP rs17252760, chrX: $148,653,916)$ and contains 5 protein-coding RefSeq genes: FMR1, FMR1NB, AFF2, IDS, and CXorf40A (fig. 2). The deletions as well as their de novo occurrence were confirmed by $\mathrm{qPCR}$, MLPA, array CGH, or FMR1 CGG-repeat analysis.

In the daughter of daughter-mother control pair 3 (POF3), qPCR on genomic DNA confirmed the microscopically visible $\operatorname{del}(\mathrm{X})(\mathrm{q} 27)$ by demonstrating a copy number of 1 for $\mathrm{qPCR}$ probes localized within FMR1 and IDS. Corresponding qPCR analyses of all other POF control samples, including her mother (POF3M), excluded deletions in all 5 amplicons tested (data not shown).

\section{FMR1 Sequence and Repeat Analyses}

For all 3 patients harboring an FMR1 deletion, sequence analysis of the entire coding region of the remaining FMR1 allele was performed and revealed no pathogenic mutations. The FMR1 CGG repeat analysis by PCR and/or Southern blotting for patients and POF controls showed normal results, except for premutation alleles identified in control daughter POF1 (31/79 repeats) and her mother POF1M (29/72 repeats).

\section{X-Inactivation Analysis}

$\mathrm{X}$-inactivation studies showed random $\mathrm{X}$-inactivation patterns for all 3 patients (patient 1, 62:38; patient 2, 57: 43; patient 3, 48:52). Control daughter POF3 showed $100 \%$ skewed XCI.

\section{Expression Analyses}

We performed gene expression studies with predesigned Taqman $^{\circledR}$ gene expression assays for FMR1, FMR1NB, AFF2, and IDS on whole blood RNA. The expression of the FMR1NB gene was too low to obtain results.

IDS transcript levels were reduced in all 3 patients compared to the mean of 5 healthy female controls, significantly $(\mathrm{p}<0.05)$ in patients 1 and 3 . All 3 patients also showed significantly reduced IDS expression compared to their respective mothers (fig. 3a). None of the POF controls showed reduced expression of IDS (data not shown). Even in POF control 3, the carrier of the cytogenetically visible del(X)(q27) including IDS with com- 


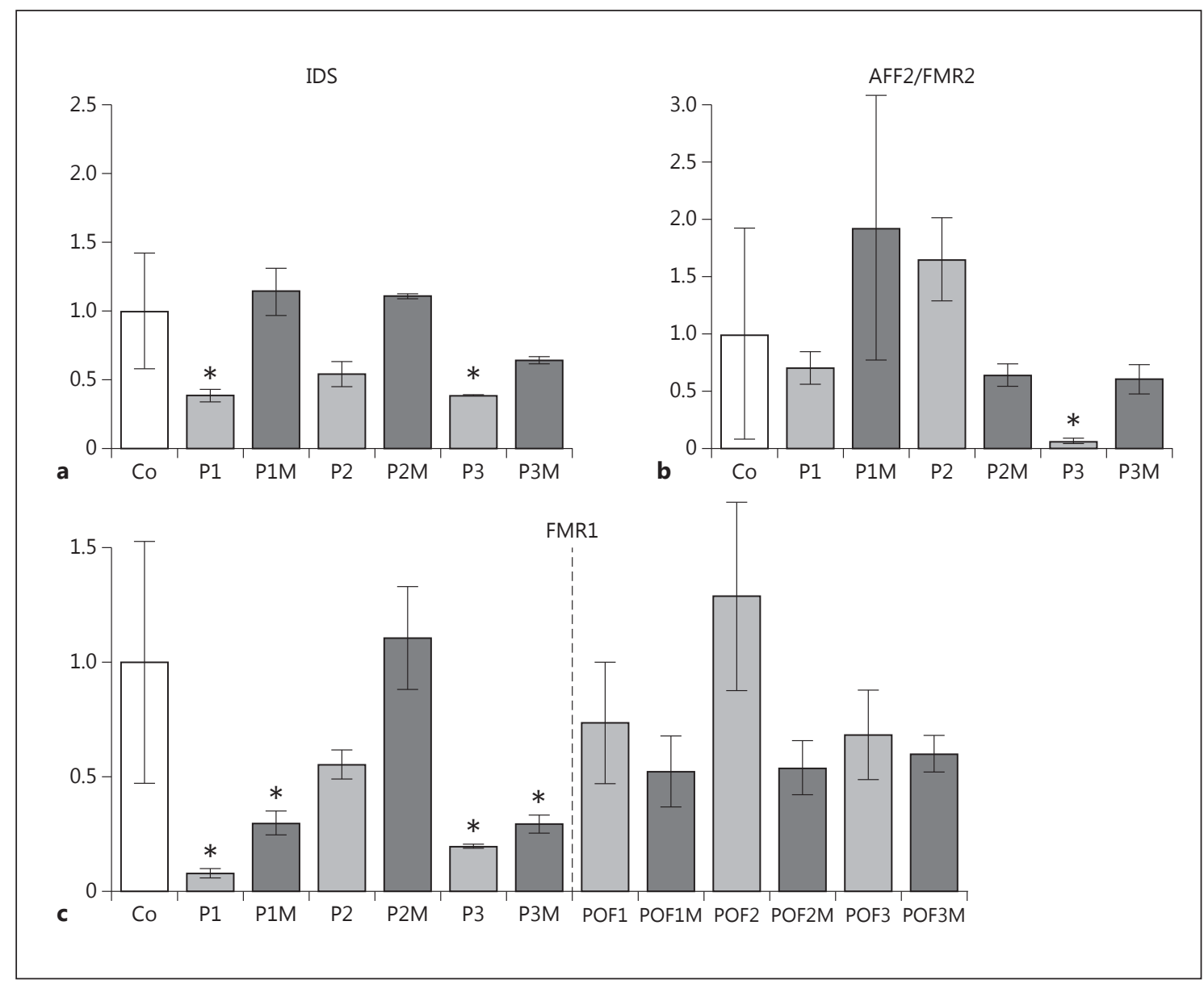

Fig. 3. Results of expression analyses of $I D S(\mathbf{a}), A F F 2$ (b) and FMR1 (c). The mean expression of 5 healthy female controls (Co) is set to 1 (white bars; error bars represent the standard deviation over all controls). The expression data of deletion patients (P1-P3) and their mothers (P1M, P2M, and P3M) as well as of POF control daughtermother pairs (POF1-POF1M, POF2-POF2M, POF3-POF3M; right of the dashed line in c) are set in relation to these 5 controls (daughters: light grey, mothers: dark grey). Error bars for patients and mothers as well as for POF control daughter-mother pairs represent the standard deviation over 2 triplicate experiments. * Expression significantly reduced compared to healthy controls.

pletely skewed X-inactivation, IDS expression levels were normal. In the POF control sample, there was also no significant difference in IDS expression between mothers and daughters (data not shown).

AFF2 transcript levels showed extreme variability with a very high standard deviation of 0.92 between the 5 healthy female controls. This high variability was also obvious in the patients and their mothers. Only patient 3 showed a significantly reduced expression of $A F F 2$ in comparison to the mean of 5 healthy controls (fig. 3b).

FMR1 transcript levels were reduced in all 3 patients in comparison to the 5 healthy female controls; in patients 1 and 3 , the difference was statistically significant (patient $1, \mathrm{p}=1.027 \times 10^{-6}$; patient $\left.3, \mathrm{p}=1.027 \times 10^{-6}\right)$. However, FMR 1 expression of the mothers of patients 1 and 3 was also significantly reduced $(\mathrm{p}=0.001023$ and $\mathrm{p}=0.000651$, respectively) (fig. 3c).

However, all 3 patients had significantly reduced FMR1 transcript levels compared to their respective mothers (patient $1, \mathrm{p}=0.001023$; patient $2, \mathrm{p}=0.002165$; patient $3, p=0.002165)$. None of the members of the POF control sample showed significantly reduced FMR1 expression, and the POF control daughters showed no reduced expression compared to their respective mothers. In addition, there was no evidence for an influence of premutations on FMR1 transcript levels: the expression pat- 
tern in POF1 and POF1M was comparable to the pattern of the other 2 control daughter-mother pairs, all of whom had normal FMR1 repeat lengths (fig. 3c).

\section{Discussion}

Here, we report 3 unrelated female patients with overlapping de novo microdeletions in Xq27.3-q28. The deletions range in size from 3.5 to $9.2 \mathrm{Mb}$ and contain 5 to 24 genes. In all 3 patients XCI was shown to be random. The 3.5-Mb SRO was identical to the deletion in patient 1 and contained the FMR1, FMR1NB, AFF2, CXorf40A, and IDS genes. Besides ID/DD, which was present in all 3 patients, patients 1 and 2 also presented with macrocephaly, muscular hypotonia, obesity, and hyperextensibility of the small joints.

To our knowledge, only 7 female index patients with ID/DD and deletions including FMR1 have been reported to date. One of these microdeletions resembled the SRO of the patients described here: the $2.7-\mathrm{Mb}$ deletion encompassed 5 genes (FMR1, FMR1NB, AFF2, IDS, and CXorf40A) and the XCI status was random (Probst et al., 2007]. This patient presented with ID/DD and obesity, as well as macrocephaly and small joint hyperextensibility; muscular hypotonia was not reported.

Among the 6 other previously reported female patients, most of the deletions were larger, and several of them additionally contained genes which cause other syndromes, complicating genotype-phenotype correlations regarding the deletions and FXS. The 3-5-cM deletion extending proximally from the $3^{\prime}$ end of the IDS gene of a severely developmentally delayed girl with skewed XCI caused a profound deficiency of IDS activity and Hunter syndrome (mucopolysaccharidosis type II/MPS II, OMIM 309900). She presented with macrocephaly (also attributable to Hunter syndrome), weight and height above the 97th percentile, dysmorphic facies, hypotonia, hepatosplenomegaly, generalized dysostosis multiplex, and mucopolysacchariduria. As opposed to the joint-mobility limitation in classical Hunter syndrome, she exhibited joint hypermobility [Clarke et al., 1991, 1992].

The third previously reported patient presented with $\mathrm{DD}$, obesity and severe hypotonia, and had a $2.71-\mathrm{Mb}$ deletion encompassing FMR1, FMR1NB, AFF2, and IDS. The probable inclusion of the X-linked myotubular myopathy gene MTM1 within the deletion could explain the severity of her hypotonia. The deleted X chromosome was active in about $80 \%$ of her leukocytes [Dahl et al., 1995]. A familial 9.3-Mb deletion in Xq26.3-q27.3 was identified in the learning-disabled and non-dysmorphic sister of the male patient 1 reported by Wolff et al. [1997], and contained 26 RefSeq genes including FMR1, FMR1NB and AFF2, but not IDS. The deleted X chromosome was active in 15-20\% of blood cells [Wolff et al., 1997]. Female patient 2 from the same study had an 8.73$\mathrm{Mb}$ deletion in Xq26.3-q27.3 including FMR1, FMR1NB, and possibly also $A F F 2$, and displayed moderate to severe ID, weight in the 97th centile, dysmorphisms including a long face and prominent chin, grand mal seizures, hypothyroidism, and a happy disposition with paroxysmal bursts of laughter; XCI was random. The sixth published patient presented with DD and language delay, obesity, seizures, and behavioral abnormalities [Nagamani et al., 2012]. Her 5.9-Mb deletion extended proximally from FMR1, and XCI was skewed (17:83). Complete skewing with preferential inactivation of the deleted $\mathrm{X}$ chromosome was detected in another patient with a $10.6-\mathrm{Mb}$ deletion encompassing FMR1, AFF2, IDS, and 110 additional RefSeq genes, who displayed global DD, mild muscular hypotonia, and growth measurements in the normal range [Marshall et al., 2013].

Additionally, there are several reports focused on male carriers of familial FMR1-containing deletions in which female carriers with different degrees of ID/DD are reported with sparse clinical data: Gedeon et al. [1992] reported the carrier mother of a son with classical FXS due to an approximately $2-\mathrm{Mb}$ deletion to have random XCI and mild intellectual impairment, and to be socially competent. IDS was included in the cytogenetically visible deletion of a mildly intellectually disabled mother and her more severely affected daughter reported by Birot et al. [1996]. Neither one presented features of Hunter syndrome, while the male proband had severe Hunter syndrome. XCI was skewed (90:10) in the mother and random in the daughter [Birot et al., 1996]. The carrier mother of a boy with severe ID and an approximately $8.5-\mathrm{Mb}$ deletion including FMR1 but not $A F F 2$ had random XCI and showed very mild mental impairment as well as normal weight and height [Parvari et al., 1999]. The report by Schmidt et al. [1990] regarding a 5-year-old girl with moderate ID, behavioral abnormalities and a cytogenetically visible deletion of Xq27 with skewed XCI is excluded from the present genotype-phenotype correlation, since the authors clearly state that it is uncertain if FMR1 is in fact deleted.

Thus, ID/DD is common to the 7 patients previously reported in the literature and the 3 patients herein with deletions including FMR1. Obesity was noted in 6 out of the 10 patients, whereas joint hyperextensibility and mac-
72

Mol Syndromol 2014;5:65-75 DOI: $10.1159 / 000357962$
Zink et al. 
rocephaly were present only in the 3 patients with the smallest deletions, encompassing FMR1, FMR1NB, AFF2, and IDS (patients 1 and 2 in this report; Probst et al. [2007]), and in the female patient reported by Clarke et al. [1991, 1992] in whom macrocephaly is also attributable to Hunter syndrome. The ID/DD, large OFC and muscular hypotonia observed in our patients are characteristic clinical features of both males and females with FXS caused by full mutations, as well as by deletions of the FMR1 gene. Hyperextensibility of the small joints, obesity and behavioral problems are also part of the phenotypic spectrum of FXS, although less common [Nowicki et al., 2007; Gallagher and Hallahan, 2012]. Additionally, copy-number changes of FMR1 have recently been proposed to cause neurodevelopmental phenotypes in the FXS clinical spectrum [Nagamani et al., 2012]. The FMR1 gene has also frequently been associated with autism spectrum disorders (ASD) [Poot, 2013]. However, the 3 patients in this report showed no signs of ASD. Since the main aspects of the patients' phenotypes could be attributed to FXS, FMR1 seems to be the most promising candidate gene within the SRO, although additional genes are deleted in all 3 patients reported here.

To explore the possible etiological involvement of additional genes in the SRO, we analyzed the expression of the 4 genes with relevant functional information: FMR1, FMR1NB, AFF2, and IDS. IDS codes for iduronate 2-sulfatase, which is deficient in patients with Hunter syndrome. IDS transcript levels were significantly reduced in all patients compared to their respective mothers and in patients 1 and 3 compared to healthy controls, while none of the control POF daughters showed significant differences compared to either the healthy controls or to their respective mothers (fig. 3a). While demonstrating the reliability of our experiments, a contribution of reduced IDS expression to the patients' phenotypes seems unlikely for 3 reasons. Firstly, our patients did not show characteristic signs of Hunter syndrome such as the typical facial features and pulmonary, liver or spleen involvement. Secondly, our patients showed random XCI, whereas the rarely described female Hunter syndrome patients with mutations in 1 copy of the IDS gene usually show extremely skewed XCI [Tuschl et al., 2005]. Thirdly, mucopolysaccharidosis was excluded by urine analysis in patient 1. For $A F F 2$, the transcript levels showed a pronounced variability both in patients and controls, with no discernible difference between groups. The transcript levels of FMR1NB in whole blood were too low to be analyzed, allowing no conclusions to be drawn concerning a possible additional etiological significance of the deletion

Deletions Including FMR1 in Female Patients with ID of this gene. Since most clinical features of our patients are part of the clinical spectrum of FXS, and since the deleted X chromosome is active in at least $38 \%$ of their cells, a significantly reduced expression as demonstrated for IDS may also have been expected for FMR1 in our patients. However, a significant reduction of FMR1 expression compared to the mean of 5 healthy female controls was only found in patients 1 and 3 . The reduction in FMR1 expression in patient 2 was not statistically significant. Furthermore, the healthy mothers of patients 1 and 3 also showed significantly reduced FMR1 transcript levels compared to the healthy female controls (fig. 3c). Intriguingly, there was a statistically significant reduction in FMR1 expression in all 3 patients relative to their mothers: FMR 1 transcript levels were reduced by $72.8 \%$ in patient 1 , by $49.8 \%$ in patient 2 , and by $26.7 \%$ in patient 3 .

To exclude the possibility of reduced $F M R 1$ expression being a frequent finding in daughters compared to their mothers, we performed similar expression studies on whole blood RNA from 3 independent control daughtermother pairs without any signs of FXS, especially ID/DD. These daughter-mother pairs had originally been ascertained because the daughters (and also mother POF2M) presented with POF. Importantly, none of these 3 control daughters showed reduced FMR1 expression compared to their mothers regardless of the presence or absence of FMR1 premutations or deletions in combination with completely skewed XCI. In fact, the maternal expression of FMR 1 was lower than in the respective daughters in all control pairs. Therefore, our results indicate that the significantly reduced FMR 1 expression in our microdeletion patients compared to their respective mothers is a specific finding, and not frequently observed in other daughter-mother pairs.

Our FMR1 expression studies presented here reproduced the previously reported variability in FMR1 expression levels and exclude an absolute threshold for FMR1 transcript levels below which ID/DD and other features of FXS are expressed [Allen et al., 2004; Schuettler et al., 2011]. However, our collective findings are consistent with the presence of an intrafamilial effect, with mRNA levels being more similar among female members of the same family than among unrelated females [García-Alegría et al., 2007]. Since in all 3 patients the significant reduction in FMR1 transcript levels compared to their respective mothers appears to be specific for their phenotype, our data may be interpreted as suggesting a family-specific 'threshold' expression level, below which an FXS phenotype may present. Due to the observed intrafamilial similarity of FMR1 mRNA levels, selection of 
appropriate controls is essential for FMR1 RNA analyses in female patients. Based on the data presented here, we suggest that closely related females (e.g. mothers) might be suitable controls.

We also speculate that the degree of relative reduction in FMR1 expression may be correlated with the severity of phenotype in our study. Patients 1 and 2 presented with a more severe phenotype and a more pronounced reduction in FMR1 expression than patient 3 (at least a $50 \%$ vs. a $27 \%$ reduction). Patients 1 and 2 also had a considerably earlier onset of ID/DD than patient 3, who was only diagnosed at the age of 2 years. Additionally, both patients 1 and 2 presented with a diminished sense of satiety resulting in obesity, muscular hypotonia and joint hyperextensibility, all of which were absent in patient 3.

We propose that the significant reduction in FMR1 expression relative to their respective mothers might play a role in the development of an FXS phenotype in female patients, in addition to the XCI status. However, several other conceivable confounding factors, such as age differences or perhaps different expression patterns of the deleted genes in other tissues or earlier in development, could not be addressed in this study and might poten- tially influence the etiology of our patients' clinical features. Further expression studies in larger patient-mother groups are needed to support this hypothesis.

In summary, we describe 3 female patients with deletions encompassing the FMR1 gene. Besides the typical signs of FXS such as ID/DD, large OFC and muscular hypotonia, 2 patients also presented with obesity, which has also been reported in 5 of 7 previously published patients [Dahl et al., 1995; Wolff et al., 1997; Probst et al., 2007]. In contrast to the control daughter-mother pairs analyzed, all 3 patients described here showed markedly reduced FMR1 expression relative to their mothers, indicating that this reduction is specific to their phenotype.

\section{Acknowledgements}

We thank the patients and their families for their kind cooperation. H.E., A.M.Z. and E.W. are members of the 'German Mental Retardation Network' (MRNET) which is funded by the German Federal Ministry of Education and Research (BMBF) as a part of the National Genome Research Network (NGFNplus/www. ngfn.de/englisch/15.htm, project reference numbers 01GS08164). We thank Ulrike Bender for her excellent technical assistance.

\section{References}

Allen EG, He W, Yadav-Shah M, Sherman SL: A Engels H, Wohlleber E, Zink A, Hoyer J, Ludwig study of the distributional characteristics of FMR1 transcript levels in 238 individuals. Hum Genet 114:439-447 (2004).

-Birot AM, Delobel B, Gronnier P, Bonnet V, Maire I, Bozon D: A 5-megabase familial deletion removes the IDS and FMR-1 genes in a male Hunter patient. Hum Mutat 7:266-268 (1996).

-Clarke JT, Greer WL, Strasberg PM, Pearce RD, Skomorowski MA, Ray PN: Hunter disease (mucopolysaccharidosis type II) associated with unbalanced inactivation of the $\mathrm{X}$ chromosomes in a karyotypically normal girl. Am J Hum Genet 49:289-297 (1991).

Clarke JT, Wilson PJ, Morris CP, Hopwood JJ, Richards RI, et al: Characterization of a deletion at Xq27-q28 associated with unbalanced inactivation of the nonmutant $\mathrm{X}$ chromosome. Am J Hum Genet 51:316-322 (1992).

DDahl N, Hu LJ, Chery M, Fardeau M, Gilgenkrantz S, et al: Myotubular myopathy in a girl with a deletion at Xq27-q28 and unbalanced $\mathrm{X}$ inactivation assigns the MTM1 gene to a $600-\mathrm{kb}$ region. Am J Hum Genet 56:11081115 (1995).

$>$ de Vries BB, Wiegers AM, Smits AP, Mohkamsing S, Duivenvoorden HJ, et al: Mental status of females with an FMR1 gene full mutation. Am J Hum Genet 58:1025-1032 (1996). gels $\mathrm{H}$, Wohlleber E, Zink A, Hoyer J, Ludwig $\mathrm{KU}$, et al: A novel microdeletion syndrome involving 5q14.3-q15: clinical and molecular cytogenetic characterization of three patients. Eur J Hum Genet 17:1592-1599 (2009).

Feng Y, Zhang F, Lokey LK, Chastain JL, Lakkis $\mathrm{L}$, et al: Translational suppression by trinucleotide repeat expansion at FMR1. Science 268: 731-734 (1995).

Fernandez-Carvajal I, Lopez Posadas B, Pan R, Raske C, Hagerman PJ, Tassone F: Expansion of an FMR1 grey-zone allele to a full mutation in two generations. J Mol Diagn 11:306-310 (2009).

Gallagher A, Hallahan B: Fragile X-associated disorders: a clinical overview. J Neurol 259:401413 (2012).

García-Alegría E, Ibáñez B, Mínguez M, Poch M, Valiente A, et al: Analysis of FMR1 gene expression in female premutation carriers using robust segmented linear regression models. RNA 13:756-762 (2007).

Gedeon AK, Baker E, Robinson H, Partington MW, Gross B, et al: Fragile X syndrome without CCG amplification has an FMR1 deletion. Nat Genet 1:341-344 (1992).

Hammond LS, Macias MM, Tarleton JC, Shashidhar Pai G: Fragile X syndrome and deletions in FMR1: new case and review of the literature. Am J Med Genet 72:430-434 (1997).
Lau AW, Brown CJ, Peñaherrera M, Langlois S, Kalousek DK, Robinson WP: Skewed X-chromosome inactivation is common in fetuses or newborns associated with confined placental mosaicism. Am J Hum Genet 61:1353-1361 (1997).

Marshall LS, Simon J, Wood T, Peng M, Owen R, et al: Deletion Xq27.3q28 in female patient with global developmental delays and skewed X-inactivation. BMC Med Genet 14:49 (2013).

- Nagamani SC, Erez A, Probst FJ, Bader P, Evans $P$, et al: Small genomic rearrangements involving FMR1 support the importance of its gene dosage for normal neurocognitive function. Neurogenetics 13:333-339 (2012).

Nowicki ST, Tassone F, Ono MY, Ferranti J, Croquette MF, et al: The Prader-Willi phenotype of fragile X syndrome. J Dev Behav Pediatr 28: 133-138 (2007).

O’Donnell WT, Warren ST: A decade of molecular studies of fragile X syndrome. Annu Rev Neurosci 25:315-338 (2002).

Parvari R, Mumm S, Galil A, Manor E, Bar-David $\mathrm{Y}$, Carmi R: Deletion of $8.5 \mathrm{Mb}$, including the $F M R 1$ gene, in a male with the fragile $\mathrm{X}$ syndrome phenotype and overgrowth. Am J Med Genet 83:302-307 (1999). 
Poot M: Towards identification of individual etiologies by resolving genomic and biological conundrums in patients with autism spectrum disorders. Mol Syndromol 4:213-226 (2013).

Probst FJ, Roeder ER, Enciso VB, Ou Z, Cooper $M L$, et al: Chromosomal microarray analysis (CMA) detects a large $\mathrm{X}$ chromosome deletion including FMR1, FMR2, and IDS in a female patient with mental retardation. Am J Med Genet A 143:1358-1365 (2007).
Schmidt M, Certoma A, Du Sart D, Kalitsis P, Leversha $\mathrm{M}$, et al: Unusual $\mathrm{X}$ chromosome inactivation in a mentally retarded girl with an interstitial deletion Xq27: implications for the fragile X syndrome. Hum Genet 84:347-352 (1990).

Schuettler J, Peng Z, Zimmer J, Sinn P, von Hagens $\mathrm{C}$, et al: Variable expression of the Fragile X Mental Retardation 1 (FMR1) gene in patients with premature ovarian failure syndrome is not dependent on number of (CGG) $\mathrm{n}$ triplets in exon 1. Hum Reprod 26:12411251 (2011).

- Tassone F, Hagerman RJ, Taylor AK, Gane LW, Godfrey TE, Hagerman PJ: Elevated levels of FMR1 mRNA in carrier males: a new mechanism of involvement in the fragile- $\mathrm{X}$ syndrome. Am J Hum Genet 66:6-15 (2000).
Tuschl K, Gal A, Paschke E, Kircher S, Bodamer OA: Mucopolysaccharidosis type II in females: case report and review of literature. Pediatr Neurol 32:270-272 (2005).

Wolff DJ, Gustashaw KM, Zurcher V, Ko L, White W, et al: Deletions in Xq26.3-q27.3 including FMR1 result in a severe phenotype in a male and variable phenotypes in females depending upon the $\mathrm{X}$ inactivation pattern. Hum Genet 100:256-261 (1997). 